\title{
Estimation of Out-of-Field Dose Variation using Markus Ionization Chamber Detector
}

\author{
Ahmed M. Abdelaal ${ }^{a^{*}}$, Ehab M. Attalla ${ }^{b}$, Wael M. Elshemey ${ }^{c}$ \\ ${ }^{a}$ Radiotherapy Department, Nasser Institute, Cairo, Egypt. \\ ${ }^{b}$ National Cancer Institute, Cairo University, Egypt. \\ ${ }^{c}$ Biophysics Department, Faculty of Science, Cairo University, Egypt. \\ Received 08 January 2020; Accepted 21 February 2020
}

\begin{abstract}
Objective: The aim of This work to provide evaluation for the out-of-field dose with different plan parameters as field size and depth using Markus ionization chamber detector in the measurement that are frequently used in electron and superficial dosimetery, in radiotherapy. Methods: This is carried out through the application of these detector in estimation of the outof-field dose with important dosimetric parameters such as field size (from $5 \times 5$ to $30 \times 30 \mathrm{~cm}^{2}$ ) and depth (from 1.5 to 30 $\mathrm{cm}$ ) at energy $6 \mathrm{MV}$ and collimator angle $0^{\circ}$ at SSD $100 \mathrm{~cm}$. Results: Results show that, the Markus detector reported an increase in out-of-field dose with field size, depth in almost all measurements. For $6 \mathrm{MV}$ and $0^{\circ}$ collimator angle, the outof-field dose at field size of $5 \times 5 \mathrm{~cm}^{2}$ (depth of $1.5 \mathrm{~cm}$ ) is $1.1 \%$ and at field size of $30 \times 30 \mathrm{~cm}^{2}$ (depth of $1.5 \mathrm{~cm}$ ) is $4.4 \%$. The out-of-field dose for a depth of $1.5 \mathrm{~cm}$ (field size of $10 \times 10 \mathrm{~cm}^{2}$ ) is $2.3 \%$ and for a depth of $30 \mathrm{~cm}$ (field size of $10 \times 10$ $\mathrm{cm}$ ) is $5.5 \%$. the measured out-of-field dose by Markus detector overestimated in the calculated at different field sizes $\left(2.7 \%\right.$ instead of $2.3 \%$ at field size of $10 \times 10 \mathrm{~cm}^{2}$ and $5.2 \%$ instead of $4.4 \%$ at field size of $\left.30 \times 30 \mathrm{~cm}^{2}\right)$ and different depths (2.7\% instead of $1.1 \%$ at depth of $1.5 \mathrm{~cm}$ and $4.1 \%$ instead of $3.4 \%$ at depth of $30 \mathrm{~cm}$ ). Analysis: The result reported an increase in mean out-of-field dose with field size, depth, energy and SSD. Markus ionization chamber detector show overestimation of the measured out-of-field dose in the calculated values at all field sizes and depths, this may be attributed to the poor detection of out-of-field dose by TPS.
\end{abstract}

Keywords: Markus; Out-of-field dose; Dosimetry; Radiotherapy.

\section{Introduction}

The aim of radiation therapy is the irradiation of a target volume while minimizing the amount of radiation that reaches normal tissue. The beam shaping is an important method to minimize the absorbed dose in normal tissue and critical structures [1].

In radiotherapy, we must consider harmful effects of radiation for healthy tissues surrounding tumor tissues [2, 3]. The normal tissues protection can be performed by either Cerrobend blocks [4] or Multi-Leaf Collimators (MLCs) [5]. Regardless of the type of radiotherapy being used, out-of-field doses delivered by photons, electrons, protons, or neutrons pose unique challenges to medical physicists. This report details many of those challenges and gives guidance to the medical physics community on how to best determine the out-of-field doses associated with modern radiotherapy techniques and modalities [6].

The occurrence of secondary malignancies in patients treated with radiation was found to be maximum in the

* Corresponding author: mosa_science2010@yahoo.com

$>$ This is an open access article under the CC-BY license (https://creativecommons.org/licenses/by/4.0/).

(C) Authors retain all copyrights. 
normal tissue surrounding the target where the highest dose was delivered [7]. An increased risk of cancer incidence after exposure to low doses has been previously reported [8, 9]. In clinical photon beams, the dose outside the geometrical field limits is produced by photons originating from head leakage, scattering at the beam collimators and the flattening filter (head scatter) and scattering from the directly irradiated region of the patient or phantom [10].

It was found that the radiation scattering from patient is the main cause of dose near the edge of treatment field, while the leakage radiation has the main contribution at large distances from the edge of treatment field [11]. A general strategy for out-of-field dose estimation requires development and improvement in several areas including (i) dosimetry in regions of steep dose gradient close to the field edge (ii) experimentally verified analytical and Monte Carlo models for out-of-field doses (iii) the validity of treatment planning system algorithms outside the field edge (iv) dosimetry of critical sub-structures in organs at risk (v) mixed field (including neutron) dosimetry in proton and ion radiotherapy and photoneutron production in high energy photon beams (vi) the most appropriate quantities to use in neutron dosimetry in a radiotherapy context and (vii) simplification of measurement methods in regions distant from the target volume [12].

An accurate assessment of the secondary cancer risk that can result from a treatment with radiation requires a detailed knowledge of the dose profile outside the tumor [13]. Any reduction in out-of-field dose to healthy tissue is therefore important for long term risk reduction, and accurate calculation of out-of-field dose to be crucial for informing risk estimates [14]. TPSs are modeling the treatment fields to deliver adequate dose to target. Many planning algorithms are developed for accurate dose predication in field dosimetry, while out-of-field dose predictions are poor $[15,16]$.

\section{Materials and Methods}

\subsection{Markus Ionization Chamber Detector}

An advanced Markus ionization chamber (model TM 34045, inner diameters of $1.3 \mathrm{~mm}$, volume of $0.02 \mathrm{~cm}^{3}$, PTW, Germany). This is a further development of the classic Markus chamber that is characterized by a wide guard ring design to avoid perturbation effects. The outer shape is identical with the classic Markus chamber. The chamber is ideal for dose distribution measurements in a water phantom where the detector sensitive volume is small due to its small surface thickness so this chamber recommended in superficial measurement (low dose). This provides a good spatial resolution. The Markus ionization chamber detector can be used in electron and photon dosimetry as reported in IAEA TRS 381 and 398.

\subsection{Three Dimensional Water Phantom}

A Large size motorized 3D water phantom (Model MP3-M, with moving detector range of $60 \times 50 \times 40.7 \mathrm{~cm}^{3}, \mathrm{PTW}$, Germany) is used for automatic dose distribution measurements of radiation therapy beams. It includes a precision 3D stainless steel movement mechanism and three stepper motors for a detector positioning speed of $50 \mathrm{~mm} / \mathrm{s}$ and a positioning accuracy of $\pm 0.1 \mathrm{~mm}$. The delivery includes a cable connection box mounted to the tank. To operate the tank, MEPHYSTO software and an electronic system (TBA) are required.

\subsection{Linear Accelerator (Linac)}

A Siemens ONCOR Linac is used in this study. It is a multi-energy machine (6 MV and $15 \mathrm{MV}$ operating up to $500 \mathrm{MU} / \mathrm{min}$ and 6 electron energies). The MLC delivery system replaces the lower movable jaws inside the linear accelerator head. The OPTIFOCUS MLC for the ONCOR linear accelerators has 41 pairs of inner leaves with a 1.0 $\mathrm{cm}$ width that is projected at isocenter.

\subsection{Treatment Planning System (TPS)}

XIO Treatment Planning System (version 4.6.3, Elekta, CMS, England) is used in this work. It include convolution, Clarkson and superposition algoritms in dose calculation for photon mode therapy and pencil beam algorithm in electron mode therapy. It uses a cumulative Dose-Volume Histogram (DVH) in plans evaluation. It has an Intensity Modulated Radiotherapy (IMRT) option. The leaf sequencer is used to convert an optimized fluence into a deliverable sequence of MLC segments.

\subsection{Irradiation Condition}

Field sizes ranging from $10 \times 10$ to $30 \times 30 \mathrm{~cm}^{2}$ are used to perform the out-of-field dose measurements at SSD of $100 \mathrm{~cm}$ and energy $6 \mathrm{MV}$. The out-of-field dose is measured at distances ranging from 1 to $12 \mathrm{~cm}$ with increment of $1 \mathrm{~mm}$ from the field edge along the $\mathrm{x}$ axis (cross plane direction). Markus ionization chamber detectors are used in the out-of-field dose measurement. The out-of-field dose is calculated as the percentage of dose on the central axis for each field size and depth to dose at a selected distance from field edge at Dmax. The out-of-field dose is measured at 
collimator angles of $0^{\circ}$. Calculated dose profiles are obtained from non-image patient study sets on TPS. The profile is sent in Ascii data format through a network to a dose profile folder in the TPS and compared to the measured profile using a Microsoft Excel data sheet. The dose measurements in the penumbra region are inaccurate, the statistical dose analysis are performed in the region below $20 \%$ dose. This allowed the evaluation of the percentage deviation of the calculated out-of-field dose from the measured one through the relation (measured-calculated)/calculated \%).

\section{Results and Discussion}

\subsection{Out-of-field Dose with Different Field Sizes}

Figure 1 shows the variation in the out-of-field dose with distance to central axis for three different field sizes $10 \times 10,20 \times 20$ and $30 \times 30 \mathrm{~cm}^{2}$, respectively at a depth of $1.5 \mathrm{~cm}$ with collimator angle of $0^{\circ}$ and an energy of $6 \mathrm{MV}$ using Markus ionization chamber detectors.

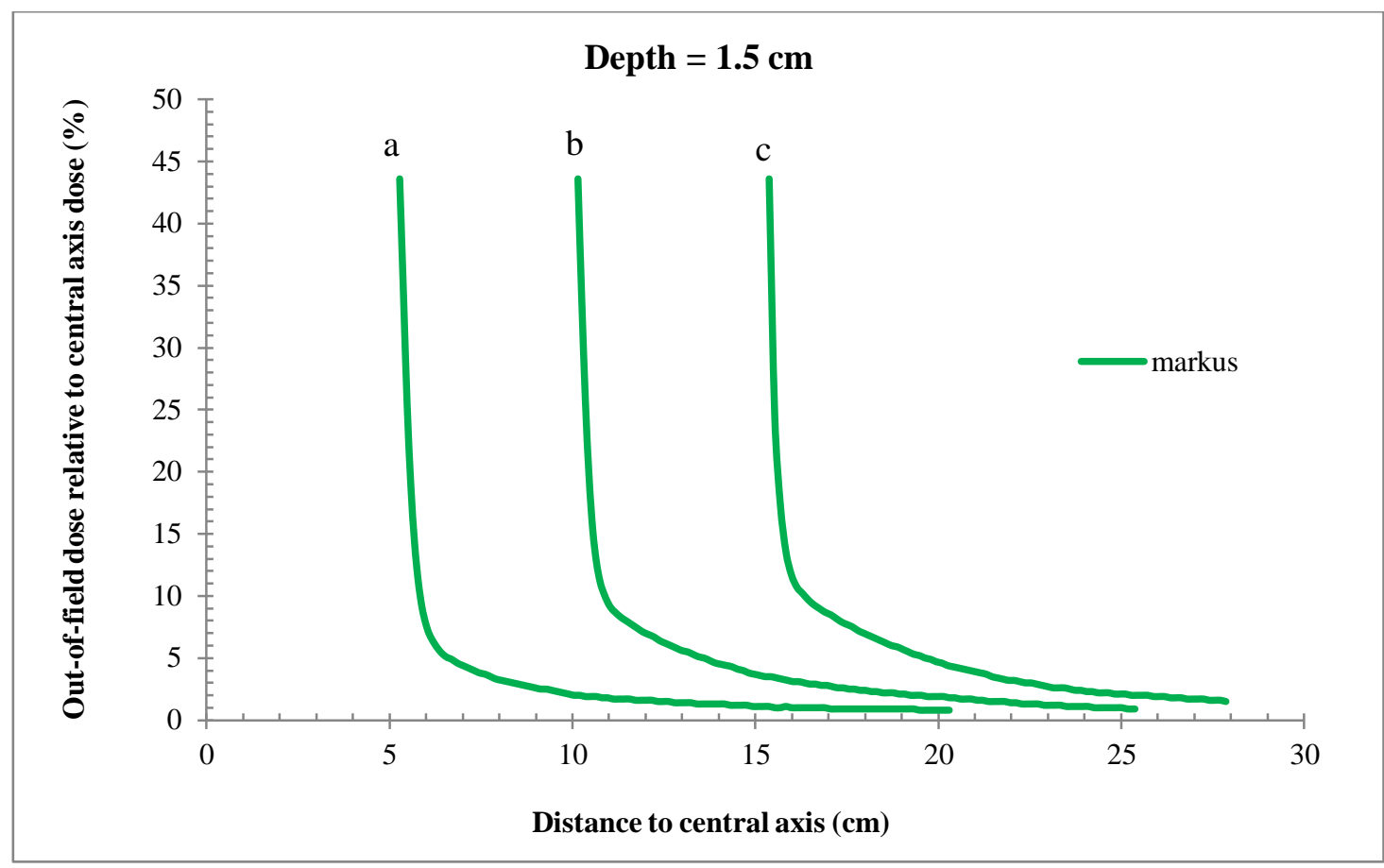

Figure 1.The variation in the out-of-field dose with distance to central axis for different field sizes a: $10 \times 10 \mathrm{~cm}^{2}, \mathrm{~b}: 20 \times 20 \mathrm{~cm}^{2}$ and c: $30 \times 30 \mathrm{~cm}^{2}$ using Markus ionization chamber detectors

Typical profile shapes can be seen with a sharp decrease in the out-of-field dose at shorter distances from the central axis followed by a small decrease at greater distances from the central axis [17]. This behaviour is because collimator scatter, phantom scatter and head leakage all contribute to the out-of-field dose at the central axis while at a distance far from the central axis, only collimator scatter and head leakage contributes to the out-of-field dose [10].

For the three given field sizes, the Markus detector exhibits higher out-of-field dose with all three field sizes. This behaviour is more apparent for the $30 \times 30 \mathrm{~cm}^{2}$ field. The mean out-of-field dose also shows higher values (Figure 2). Moreover, one can notice that there is always an increase in the mean out-of-field dose with increase in field size. 


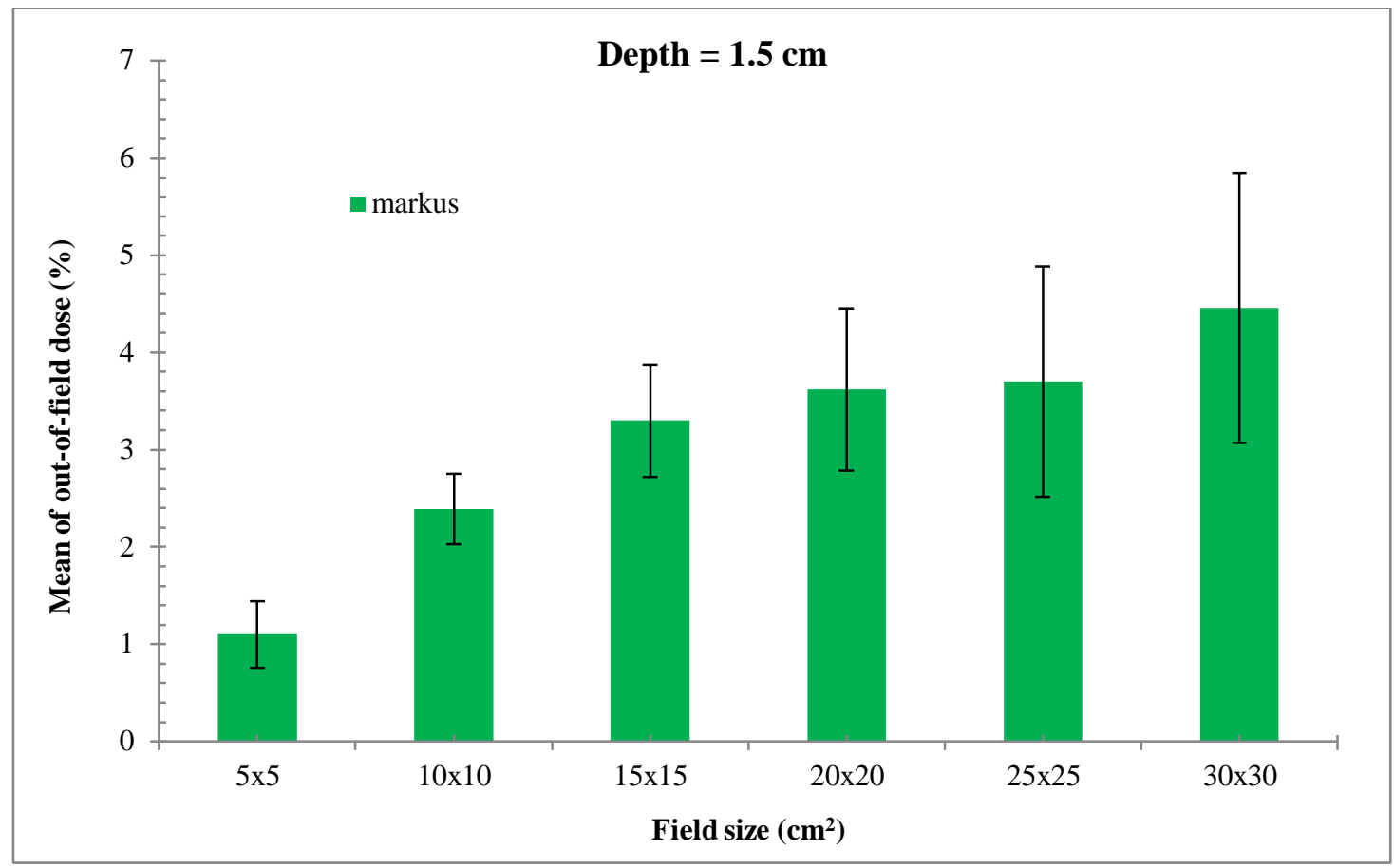

Figure 2.Variation of mean out-of-field dose with different field sizes using Markus ionization chamber detectors. The error bars represent the standard deviation (SD)

At a depth of $1.5 \mathrm{~cm}$, the mean out-of-field dose increases from 1.1 to $4.4 \%$ at field size of $5 \times 5$ and $30 \times 30 \mathrm{~cm}^{2}$, respectively. This variation in out- of- field dose with different field size especially at larger field size can explain through that, at large field sizes, the interaction of x-ray photons with larger area of the linear accelerator head parts results in a considerable increase in the scattering volume defined by collimator size hence this lead to the patient scatter increase, collimator scatter and patient scatter are important components in out-of-field dose. This explains the observed increase in the mean out-of-field dose with the increase in field size $[18,19]$.

\subsection{Out-of-field Dose with Different Depths}

Figures $3 \mathrm{a}$ and $3 \mathrm{~b}$ shows an example of the variation in the out-of-field dose with distance to central axis for a field size of $10 \times 10 \mathrm{~cm}^{2}$ at a depth of 15 and $30 \mathrm{~cm}$ using Markus ionization chamber detectors.

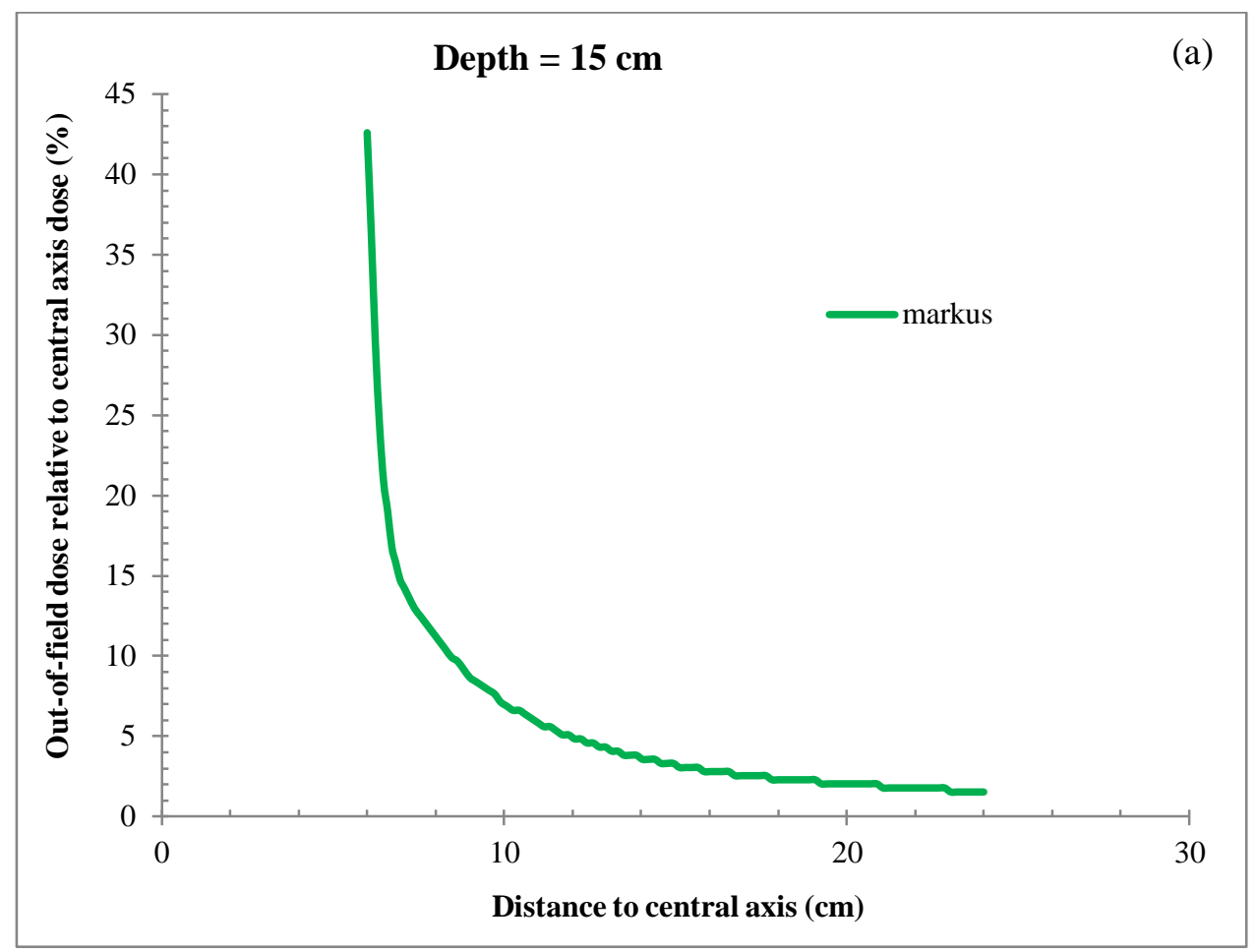




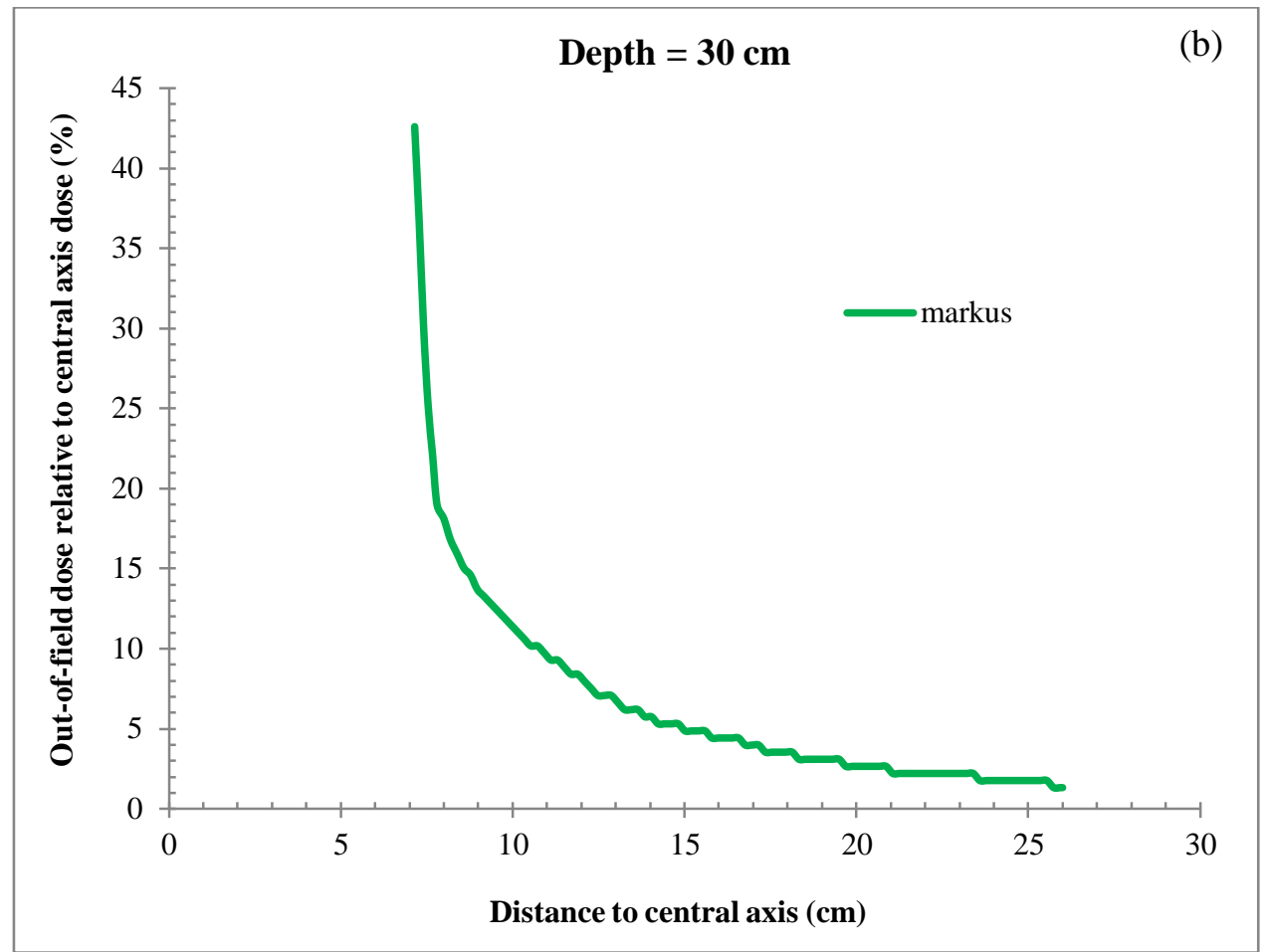

Figure 3. Variation in the out-of-field dose with distance to central axis for field size $10 \times 10 \mathrm{~cm}^{2}$ at a depth of: a) $15 \mathrm{~cm}$ and b) $30 \mathrm{~cm}$ using Markus ionization chamber detectors

From Figure 3, the Markus detector yields greater values of out-of-field dose with the depth increase. This is more obvious in Figure 4. At a field size of $10 \times 10 \mathrm{~cm}^{2}$, the mean out-of-field dose increases from 2.3 to $5.5 \%$ at a depth of $30 \mathrm{~cm}$. There is always an increase in the mean out-of-field dose with increase in depth.

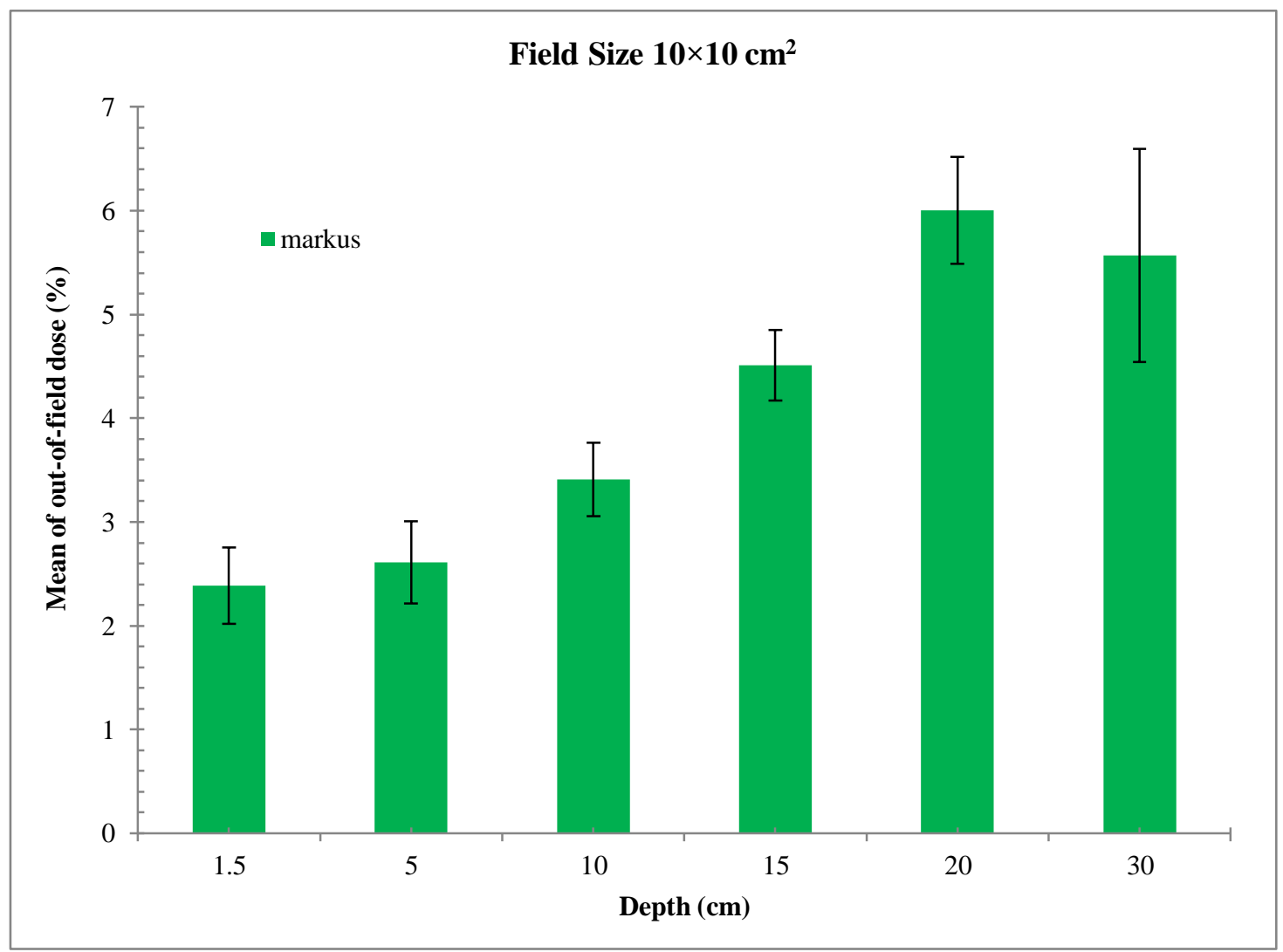

Figure 4. Variation of out-of-field dose with different depths $(1.5,5,10,15,20$ and $30 \mathrm{~cm})$ using Markus ionization chamber detectors 
The variation of out-of-field dose with the depth increase can be explained by that the cone beam geometry and dimension of irradiated field both increase with depth. This explains the corresponding increase in the amount of scattered radiation with the depth increase that contributing to the out-of-field dose at greater depths [17, 19].

\subsection{Measured and Calculated Out-of-field Dose at Different Field Sizes}

Using the Markus detector in the measurement, (Figure 4) measured out-of-field dose exhibit lower values compared to the calculated at all field sizes, this is more clear at field size $30 \times 30 \mathrm{~cm}^{2}$ Moreover, with Markus chamber detector, one can notice that there is always an increase in both measured and calculated mean out-of-field dose with field size. The measured mean out-of-field dose by Markus detector overestimated in the calculated at different field sizes by $14 \%(2.7 \%$ instead of $2.3 \%)$ at field size of $10 \times 10 \mathrm{~cm}^{2}$ and by $15 \%(5.2 \%$ instead of $4.4 \%)$ at field size of $30 \times 30 \mathrm{~cm}^{2}$. Measured and calculated doses decreased approximately exponentially as a function of distance from the field edge at peripheral regions, comparison of measured doses and doses calculated treatment planning system (TPS) showed that the TPS underestimated out-of-field dose both for IMRT and 3D-CRT [20].

Pinnacle TPS underestimates the out of field dose by an average of $50 \%$ for intensity modulated radiation therapy (IMRT) treatment plans and this underestimation is worsened with increasing distance from the edge of treatment field. While Eclipse TPS underestimated out of field doses by an average of $40 \%$ [11].

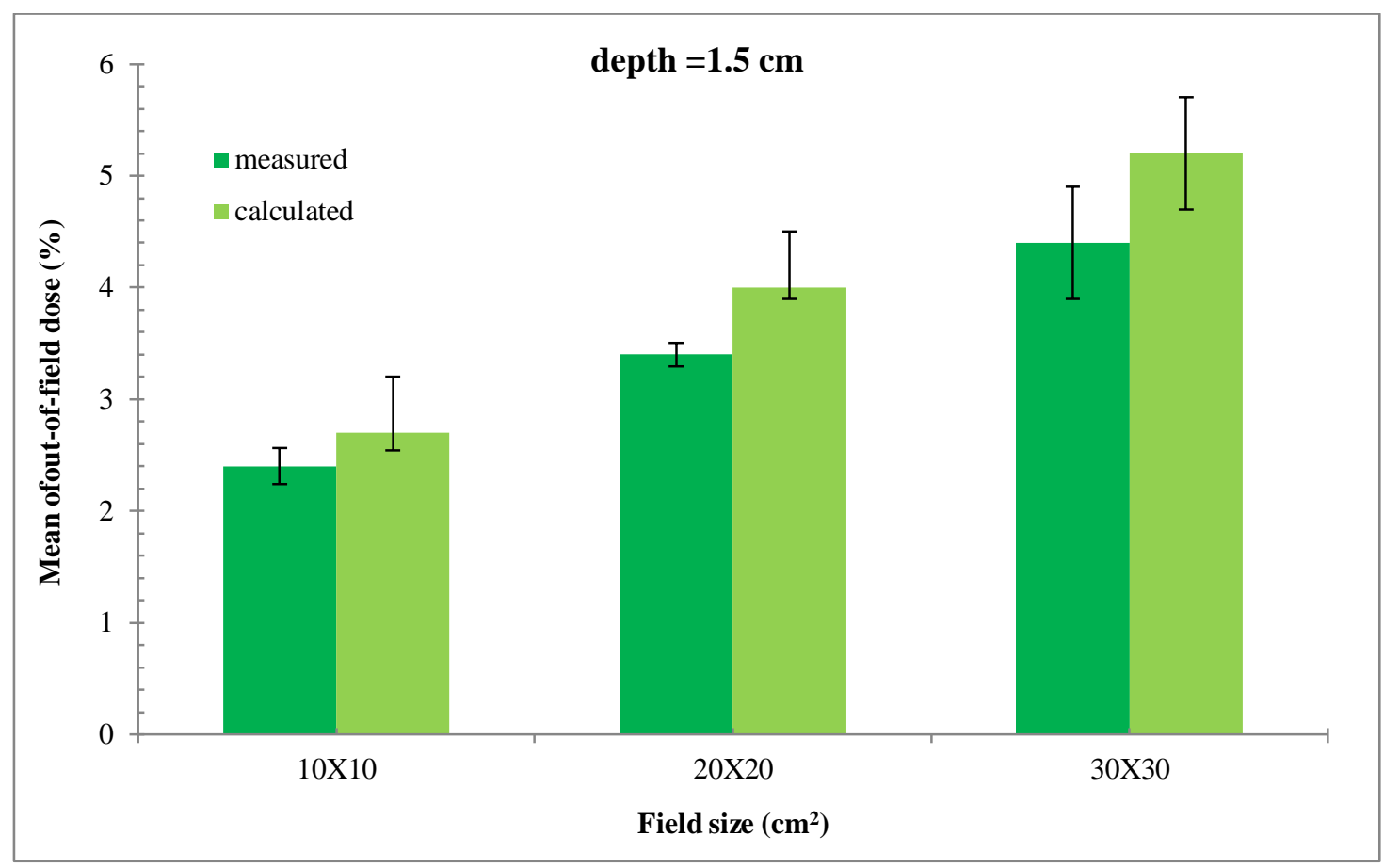

Figure 5.Variation of measured and calculated mean out-of-field dose with field size using Markus ionization chamber detector. The error bars represent the standard deviation (SD)

\subsection{Measured and Calculated Out-of-field Dose at Different Depths}

Figure 5 shows Variation of measured and calculated mean out-of-field dose with depth using Markus ionization chamber detector. Similar to the variation with field size, there is greater measured mean out-of-field dose for all depths compared to calculated using Markus ionization chamber in the measurement. Mean out-of-field dose is constantly overestimated in the calculated values for all depths by $59 \%(2.7 \%$ instead of $1.1 \%)$ at depth of $1.5 \mathrm{~cm}$ and by $17 \%(4.1 \%$ instead of $3.4 \%)$ at depth of $30 \mathrm{~cm}$.

\section{Conclusion}

The result reported an increase in mean out-of-field dose with field size, depth using markus ionization chamber detector in the measurement which are important plan parameters should be consider in design of any treatment plan. So it is important to consider the out-of-field dose with the previous parameters especially in the treatment cases with OARs near to the field edge and almost we ignore the out-field dose reach to these organ risk although this low dose can cause secondary malignancy.

The markus ionization chamber detector show overestimation of the measured out-of-field dose in the calculated values at all field sizes and depths, this may be attributed to the poor detection of out-of-field dose by TPS where there 
some of dosimetric parameter as head leakage which is one of important components that cause the out-of-field dose, we can accurate estimate the head leakage from the treatment machine using different methods as large ionization chamber detector and dosimetric films but the planning system cannot model and estimate this head leakage in accurate manner.

\section{Declaration of Competing Interest}

The authors declare that they have no known competing financial interests or personal relationships that could have appeared to influence the work reported in this paper.

\section{Ethical Approval}

All procedures performed in studies involving human participants were in accordance with the ethical standards of the institutional and/or national research committee and with the 1964 Helsinki declaration and its later amendments or comparable ethical standards.

\section{References}

[1] Jeraj, M. and Robar, V., 2004. Multileaf collimator in radiotherapy. Radiology and Oncology, 38(3):235-240+248.

[2] Johns HE and Cunningham JR., 1983. The Physics of Radiology. Radiology, 152(1), pp.194-194.

[3] Khan FM (2010). The physics of radiation therapy. 4th ed., Philadelphia: Lippincott Williams \& Wilkins.

[4] Wojcicka, J. B., Yankelevich, R., Werner, B. L., \& Lasher, D. E. (2008). Technical Note: On Cerrobend shielding for 18 22MeV electron beams. Medical Physics, 35(10), 4625-4629. doi:10.1118/1.2977801.

[5] Hogstrom, K. R., Boyd, R. A., Antolak, J. A., Svatos, M. M., Faddegon, B. A., \& Rosenman, J. G. (2004). Dosimetry of a prototype retractable eMLC for fixed-beam electron therapy. Medical Physics, 31(3), 443-462. doi:10.1118/1.1644516.

[6] Kry, S. F., Bednarz, B., Howell, R. M., Dauer, L., Followill, D., Klein, E., ... George Xu, X. (2017). AAPM TG 158: Measurement and calculation of doses outside the treated volume from external-beam radiation therapy. Medical Physics, 44(10), e391-e429. doi:10.1002/mp.12462.

[7] Dörr, W., \& Herrmann, T. (2002). Cancer induction by radiotherapy: dose dependence and spatial relationship to irradiated volume. Journal of Radiological Protection, 22(3A), A117-A121. doi:10.1088/0952-4746/22/3a/321.

[8] Pierce, D. A., \& Preston, D. L. (2000). Radiation-related cancer risks at low doses among atomic bomb survivors. Radiation research, 154(2), 178-186. doi:10.1667/0033-7587(2000)154[0178:RRCRAL]2.0.CO;2.

[9] Preston, D. L., Shimizu, Y., Pierce, D. A., Suyama, A., \& Mabuchi, K. (2003). Studies of Mortality of Atomic Bomb Survivors. Report 13: Solid Cancer and Noncancer Disease Mortality: 1950-1997. Radiation Research, 160(4), $381-407$. doi:10.1667/rr3049.

[10] Chofor, N., Harder, D., Willborn, K. C., \& Poppe, B. (2012). Internal scatter, the unavoidable major component of the peripheral dose in photon-beam radiotherapy. Physics in Medicine and Biology, 57(6), 1733-1743. doi:10.1088/00319155/57/6/1733.

[11] Farhood, B., \& Ghorbani, M. (2019). Dose calculation accuracy of radiotherapy treatment planning systems in out-of-field regions. Journal of Biomedical Physics \& Engineering, 9(2), 133.

[12] Harrison, R. (2017). Out-of-field doses in radiotherapy: Input to epidemiological studies and dose-risk models. Physica Medica, 42, 239-246. doi:10.1016/j.ejmp.2017.02.001.

[13] Xu, X. G., Bednarz, B., \& Paganetti, H. (2008). A review of dosimetry studies on external-beam radiation treatment with respect to second cancer induction. Physics in Medicine and Biology, 53(13), R193-R241. doi:10.1088/0031-9155/53/13/r01.

[14] Lonski, P., Kron, T., Taylor, M., Phipps, A., Franich, R., \& Chua, B. (2018). Assessment of leakage dose in vivo in patients undergoing radiotherapy for breast cancer. Physics and Imaging in Radiation Oncology, 5, 97-101. doi:10.1016/j.phro.2018.03.004.

[15] Jang, S. Y., Liu, H. H., \& Mohan, R. (2008). Underestimation of Low-Dose Radiation in Treatment Planning of IntensityModulated Radiotherapy. International Journal of Radiation Oncology*Biology*Physics, 71(5), $1537-1546$. doi:10.1016/j.ijrobp.2008.04.014.

[16] Kaderka, R., Schardt, D., Durante, M., Berger, T., Ramm, U., Licher, J., \& Tessa, C. L. (2012). Out-of-field dose measurements in a water phantom using different radiotherapy modalities. Physics in Medicine and Biology, 57(16), 50595074. doi:10.1088/0031-9155/57/16/5059. 
[17] Vlachopoulou, V., Malatara, G., Delis, H., Theodorou, K., Kardamakis, D. and Panayiotakis, G., (2010). Peripheral dose measurement in high-energy photon radiotherapy with the implementation of MOSFET. World Journal of Radiology, 2(11), 434. doi:10.4329/wjr.v2.i11.434.

[18] Taylor, M., \& Kron, T. (2011). Consideration of the radiation dose delivered away from the treatment field to patients in radiotherapy. Journal of Medical Physics, 36(2), 59. doi:10.4103/0971-6203.79686.

[19] Annamalai, G., \& Velayudham, R. (2009). Comparison of peripheral dose measurements using Ionization chamber and MOSFET detector. Reports of Practical Oncology \& Radiotherapy, 14(5), 176-183. doi:10.1016/s1507-1367(10)60033-8

[20] Majer, M., Stolarczyk, L., De Saint-Hubert, M., Kabat, D., Knežević, Ž., Miljanić, S., ... Harrison, R. (2017). Out-of-field Dose Measurements For 3D Conformal and Intensity Modulated Radiotherapy of a Paediatric Brain Tumour. Radiation Protection Dosimetry, 176(3), 331-340. doi:10.1093/rpd/ncx015. 